International Journal of Pure and Applied Mathematics

Volume 84 No. 5 2013, 451-461

ISSN: 1311-8080 (printed version); ISSN: 1314-3395 (on-line version)

url: http://www.ijpam.eu

doi: http://dx.doi.org/10.12732/ijpam.v84i5.1

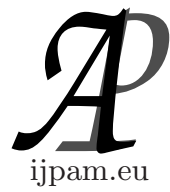

\title{
A NEW ONE PARAMETER FAMILY OF ITERATIVE METHODS WITH EIGHTH-ORDER OF CONVERGENCE FOR SOLVING NONLINEAR EQUATIONS
}

\author{
Hani I. Siyyam ${ }^{1 \S}$, Mohd Taib. Shatnawi ${ }^{2}$, I.A. Al-Subaihi ${ }^{3}$ \\ ${ }^{1,3}$ Department of Mathematics \\ Faculty of Science \\ Taibah University \\ Almadinah Almanwarra, KINGDOM OF SAUDI ARABIA \\ ${ }^{2}$ Al-Huson University College \\ Al-Balqa Applied University \\ Irbid, JORDAN
}

\begin{abstract}
In this paper, a new one parameter family of iterative methods with eighth-order of convergence for solving nonlinear equations is presented and analyzed. This new family of iterative methods is obtained by composing an iterative method proposed by Chun [3] with Newton's method and approximating the first-appeared derivative in the last step by a combination of already evaluated function values. The proposed family is optimal since its efficiency index is $8^{1 / 4} \approx 1.6818$. The convergence analysis of the new family is studied in this paper. Several numerical examples are presented to illustrate the efficiency and accuracy of the family.
\end{abstract}

AMS Subject Classification: 41A25, 65H05

Key Words: Newton's method, iterative methods, efficiency index, order of convergence, optimal eighth-order

Received: July 26, 2011

(c) 2013 Academic Publications, Ltd. url: www.acadpubl.eu

$\S_{\text {Correspondence author }}$ 


\section{Introduction}

In recent years, many researchers have been paid attention to develop several two-step, three-step and fourth-step iterative methods for solving nonlinear equations $f(x)=0$, where $f: I \subseteq R \rightarrow R$ is a real and sufficiently smooth function in $I$, with $I$ a real open interval with the assumption that $f$ has a simple zero at $\alpha$ in $I$, i.e., $f(\alpha)=0$ and $f^{\prime}(\alpha) \neq 0$. These methods have been constructed using different techniques, see [1-20].

One of the powerful methods for solving nonlinear equation of the form

$$
f(x)=0,
$$

is the Newton's method which given as comes next:

$$
x_{n+1}=x_{n}-\frac{f\left(x_{n}\right)}{f^{\prime}\left(x_{n}\right)}
$$

which has a quadratic convergence.

Chun in [3] proposed the following family of iterative methods:

$$
\begin{gathered}
y_{n}=x_{n}-\frac{f\left(x_{n}\right)}{f^{\prime}\left(x_{n}\right)} \\
z_{n}=x_{n}+(1+\beta) \frac{f\left(x_{n}\right)+f\left(y_{n}\right)}{f^{\prime}\left(x_{n}\right)}-2 \frac{f^{2}\left(x_{n}\right)}{f^{\prime}\left(x_{n}\right)\left(f\left(x_{n}\right)-f\left(y_{n}\right)\right)} \\
-\beta\left(\frac{f\left(x_{n}\right)}{f^{\prime}\left(x_{n}\right)}+\frac{f^{\prime}\left(x_{n}\right) f\left(y_{n}\right)}{f^{2}\left(x_{n}\right)+f^{\prime} 2\left(x_{n}\right)}\right),
\end{gathered}
$$

where $\beta$ is any real number for solving equation (1). It has proven in [3] that the order of convergence of this family is four and the error equation is

$$
e_{n+1}=\left(\beta c_{2}+3 c_{2}^{3}-c_{2} c_{3}\right) e_{n}^{4}+O\left(e_{n}^{5}\right) .
$$

Now by composing the above family with the classical Newton's method

$$
x_{n+1}=z_{n}-\frac{f\left(z_{n}\right)}{f^{\prime}\left(z_{n}\right)},
$$

we obtain the following family of three-step iterative methods

$$
y_{n}=x_{n}-\frac{f\left(x_{n}\right)}{f^{\prime}\left(x_{n}\right)}
$$




$$
\begin{gathered}
z_{n}=x_{n}+(1+\beta) \frac{f\left(x_{n}\right)+f\left(y_{n}\right)}{f^{\prime}\left(x_{n}\right)}-2 \frac{f^{2}\left(x_{n}\right)}{f^{\prime}\left(x_{n}\right)\left(f\left(x_{n}\right)-f\left(y_{n}\right)\right)} \\
-\beta\left(\frac{f\left(x_{n}\right)}{f^{\prime}\left(x_{n}\right)}+\frac{f^{\prime}\left(x_{n}\right) f\left(y_{n}\right)}{f^{2}\left(x_{n}\right)+f^{\prime 2}\left(x_{n}\right)}\right), \\
x_{n+1}=z_{n}-\frac{f\left(z_{n}\right)}{f^{\prime}\left(z_{n}\right)} .
\end{gathered}
$$

According to the following theorem, which can be found in [20].

Theorem 1. Let $\phi_{1}(x)$ and $\phi_{2}(x)$ be two iterative methods with order of convergence $p$ and $q$, respectively, then the order of convergence of the iterative method $\phi(x)=\phi_{2}\left(\phi_{1}(x)\right)$ is $p q$.

The order of convergence of the family (5) is eight. Per one iteration, any member in this family requires three evaluations of the function and two evaluations of its first derivative, so its efficiency index is $8^{1 / 5} \approx 1.5157$, where the efficiency index of a method is defined to $\rho^{1 / \theta}$, with $\rho$ is the order of convergence and $\theta$ is the total number of evaluations of the function and its derivative. The important issue here is "can we keep the order of convergence and the efficiency index increase as much as possible?". To improve the efficiency index, several estimations for the first-appeared derivative in the last step $f^{\prime}\left(z_{n}\right)$ by a combination of already evaluated function values are proposed, see, for example $[5,10]$.

In this paper, another estimation for $f^{\prime}\left(z_{n}\right)$ is proposed by a combination of already evaluated function values. The derivation of the estimation and construction of the new family of iterative methods will be discussed in Section 2. The analysis convergence of the proposed family will be analyzed in Section 3. Several numerical examples are given and compared with other iterative methods of the same order in Section 4 to illustrate the efficiency and the accuracy of the proposed family of iterative methods. Finally, some conclusions are pointed in Section 5 .

\section{Construction of a One Parameter Family of Iterative Methods with Eighth-Order of Convergence}

To construct our one parameter family of iterative methods for solving nonlinear equation (1), consider the family of iterative methods (5). To derive the estimation of $f^{\prime}\left(z_{n}\right)$, define the third degree polynomial

$$
P_{3}(x)=f\left(x_{n}\right)+f\left[x_{n}, x_{n}\right]\left(x-x_{n}\right)+f\left[x_{n}, x_{n}, y_{n}\right]\left(x-x_{n}\right)^{2}
$$




$$
+f\left[x_{n}, x_{n}, y_{n}, z_{n}\right]\left(x-x_{n}\right)^{2}\left(x-y_{n}\right),
$$

where $f\left[x_{n}, x_{n}\right]=f^{\prime}\left(x_{n}\right), f\left[x_{n}, y_{n}, z_{n}\right]=\frac{f\left[y_{n}, z_{n}\right]-f\left[x_{n}, y_{n}\right]}{\left(z_{n}-x_{n}\right)}$ and $f\left[x_{n}, x_{n}, y_{n}, z_{n}\right]=$ $\frac{f\left[x_{n}, y_{n}, z_{n}\right]-f\left[x_{n}, x_{n}, y_{n}\right]}{\left(z_{n}-x_{n}\right)}$. With a little simplification, the polynomial $P_{3}(x)$ can be written as:

$$
\begin{aligned}
P_{3}(x)= & f\left(x_{n}\right)+f^{\prime}\left(x_{n}\right)\left(x-x_{n}\right)+f\left[x_{n}, x_{n}, y_{n}\right]\left(x-x_{n}\right)^{2}\left[1-\frac{\left(x-y_{n}\right)}{\left(z_{n}-x_{n}\right)}\right] \\
& +f\left[x_{n}, y_{n}, z_{n}\right] \frac{\left(x-x_{n}\right)^{2}\left(x-y_{n}\right)}{\left(z_{n}-x_{n}\right)}
\end{aligned}
$$

Now, it is easy to verify that $P_{3}\left(x_{n}\right)=f\left(x_{n}\right), P_{3}\left(y_{n}\right)=f\left(y_{n}\right), P_{3}\left(z_{n}\right)=f\left(z_{n}\right)$ and $P_{3}^{\prime}\left(x_{n}\right)=f^{\prime}\left(x_{n}\right)$. Therefore, this polynomial interpolates $f$ at $x_{n}, y_{n}, z_{n}$ and also $f^{\prime}\left(x_{n}\right)=P_{3}^{\prime}\left(x_{n}\right)$. By differentiating $P_{3}(x)$ and substituting in $z_{n}$, we get:

$$
\begin{aligned}
P_{3}^{\prime}\left(z_{n}\right) & =f^{\prime}\left(x_{n}\right)+\left(f\left[x_{n}, y_{n}, z_{n}\right]+f\left[x_{n}, x_{n}, y_{n}\right]\right)\left(z_{n}-x_{n}\right) \\
& +2\left(f\left[x_{n}, y_{n}, z_{n}\right]-f\left[x_{n}, x_{n}, y_{n}\right]\right)\left(z_{n}-y_{n}\right) .
\end{aligned}
$$

We approximate $f^{\prime}\left(z_{n}\right)$ which will be denoted as $p_{f}\left(z_{n}\right)$ by the following formula

$$
\begin{aligned}
f^{\prime}\left(z_{n}\right) \approx & f^{\prime}\left(x_{n}\right)+\left(f\left[x_{n}, y_{n}, z_{n}\right]+f\left[x_{n}, x_{n}, y_{n}\right]\right)\left(z_{n}-x_{n}\right) \\
& +2\left(f\left[x_{n}, y_{n}, z_{n}\right]-f\left[x_{n}, x_{n}, y_{n}\right]\right)\left(z_{n}-y_{n}\right) \\
= & p_{f}\left(z_{n}\right) .
\end{aligned}
$$

Substitute (9) into (5) to obtain the new one parameter family of iterative methods.

$$
\begin{gathered}
y_{n}=x_{n}-\frac{f\left(x_{n}\right)}{f^{\prime}\left(x_{n}\right)} \\
z_{n}=x_{n}+(1+\beta) \frac{f\left(x_{n}\right)+f\left(y_{n}\right)}{f^{\prime}\left(x_{n}\right)}-2 \frac{f^{2}\left(x_{n}\right)}{f^{\prime}\left(x_{n}\right)\left(f\left(x_{n}\right)-f\left(y_{n}\right)\right)} \\
-\beta\left(\frac{f\left(x_{n}\right)}{f^{\prime}\left(x_{n}\right)}+\frac{f^{\prime}\left(x_{n}\right) f\left(y_{n}\right)}{f^{2}\left(x_{n}\right)+f^{\prime} 2\left(x_{n}\right)}\right), \\
x_{n+1}=z_{n}-\frac{f\left(z_{n}\right)}{p_{f}\left(z_{n}\right)} .
\end{gathered}
$$

In the next section, we will prove that the family (10) has order of convergence eight for any $\beta \in R$. Per one iteration, any member of this family requires 
three evaluations of the function and one evaluation of its first derivative so its efficiency index is $8^{1 / 4} \approx 1.6818$, which implies that the efficiency index of the family of iterative methods (10) is optimal according to Kung and Trabu's conjecture [8].

\section{Convergence Analysis}

The convergence analysis of the family of three-step iterative methods (10) for solving equation (1) will be established in this section.

Theorem 2. Let $\alpha \in I$ be a simple zero of a sufficiently differentiable function $f: I \subseteq R \rightarrow R$ for an open interval $I$. If $x_{0}$ is sufficiently close to $\alpha$, then the family of iterative methods (10) has eighth-order of convergence.

Proof. Let $\alpha$ be a simple zero of equation (1) and $x_{n}=\alpha+e_{n}$. By Taylor expansion, we have

$$
\begin{gathered}
f\left(x_{n}\right)=f^{\prime}(\alpha)\left[e_{n}+c_{2} e_{n}^{2}+c_{3} e_{n}^{3}+c_{4} e_{n}^{4}+c_{5} e_{n}^{5}+c_{6} e_{n}^{6}+c_{7} e_{n}^{7}+c_{8} e_{n}^{8}+\boldsymbol{O}\left(e_{n}^{9}\right)\right], \\
f^{\prime}\left(x_{n}\right)=f^{\prime}(\alpha)\left[1+2 c_{2} e_{n}+3 c_{3} e_{n}^{2}+4 c_{4} e_{n}^{3}+5 c_{5} e_{n}^{4}+\cdots+\boldsymbol{O}\left(e_{n}^{8}\right)\right],
\end{gathered}
$$

where $c_{k}=\frac{f^{(k)}(\alpha)}{k ! f^{\prime}(\alpha)}, k=2,3, \ldots$.

Dividing (11) by (12), gives us

$$
\begin{aligned}
\frac{f\left(x_{n}\right)}{f^{\prime}\left(x_{n}\right)}= & e_{n}-c_{2} e_{n}^{2}+\left(-2 c_{3}+2 c_{2}^{2}\right) e_{n}^{3}+\left(-3 c_{4}+7 c_{2} c_{3}-4 c_{2}^{3}\right) e_{n}^{4}+\left(10 c_{2} c_{4}\right. \\
& \left.-4 c_{5}+6 c_{3}^{2}-20 c_{3} c_{2}^{2}+8 c_{2}^{4}\right) e_{n}^{5}+\cdots+\boldsymbol{O}\left(e_{n}^{9}\right) .
\end{aligned}
$$

Substituting the last equation into $y_{n}$ in (10), we have:

$$
\begin{aligned}
y_{n}= & \alpha+c_{2} e_{n}^{2}+\left(+2 c_{3}-2 c_{2}^{2}\right) e_{n}^{3}+\left(+3 c_{4}-7 c_{2} c_{3}+4 c_{2}^{3}\right) e_{n}^{4}+\left(-10 c_{2} c_{4}\right. \\
& \left.+4 c_{5}+6 c_{3}^{2}+20 c_{3} c_{2}^{2}-8 c_{2}^{4}\right) e_{n}^{5}+\cdots+\boldsymbol{O}\left(e_{n}^{9}\right) .
\end{aligned}
$$

Expanding $f\left(y_{n}\right)$ about $\alpha$ to get

$$
\begin{aligned}
f\left(y_{n}\right)= & f^{\prime}(\alpha)\left[c_{2} e_{n}^{2}+\left(2 c_{3}-2 c_{2}^{2}\right) e_{n}^{3}+\left(3 c_{4}-7 c_{2} c_{3}+5 c_{2}^{3}\right) e_{n}^{4}+\left(-10 c_{2} c_{4}\right.\right. \\
& \left.\left.+4 c_{5}-6 c_{3}^{2}+24 c_{3} c_{2}^{2}-12 c_{2}^{4}\right) e_{n}^{5}+\cdots+\boldsymbol{O}\left(e_{n}^{9}\right)\right] .
\end{aligned}
$$

Substituting (11), (12) and (15) into $z_{n}$ in (10), and simplifying to get

$$
z_{n}=\alpha+\left(-c_{2} c_{3}+3 c_{2}^{3}+c_{2} \beta\right) e_{n}^{4}+\left(-2 c_{2} c_{4}-18 c_{2}^{4}-2 c_{3}^{2}+20 c_{3} c_{2}^{2}+2 \beta c_{3}\right.
$$




$$
\left.-6 \beta c_{2}^{2}\right) e_{n}^{5}+\cdots+\boldsymbol{O}\left(e_{n}^{9}\right)
$$

Expanding $f\left(z_{n}\right)$ about $\alpha$, to get

$$
\begin{aligned}
f\left(z_{n}\right)= & f^{\prime}(\alpha)\left[\left(-c_{2} c_{3}+3 c_{2}^{3}+c_{2} \beta\right) e_{n}^{4}+\left(-2 c_{2} c_{4}-18 c_{2}^{4}-2 c_{3}^{2}+20 c_{3} c_{2}^{2}\right.\right. \\
& \left.\left.+2 \beta c_{3}-6 c_{2}^{2} \beta\right) e_{n}^{5}+\cdots+\boldsymbol{O}\left(e_{n}^{9}\right)\right] .
\end{aligned}
$$

The expressions $f\left[x_{n}, y_{n}, z_{n}\right]$ and $\left[f\left[x_{n}, x_{n}, y_{n}\right]\right.$ can be written in terms of $e_{n}$ as:

$$
\begin{aligned}
f\left[x_{n}, y_{n}, z_{n}\right]= & f^{\prime}(\alpha)\left[c_{2}+c_{3} e+\left(c_{4}+c_{2} c_{3}\right) e_{n}^{2}+\left(c_{2} c_{4}+c_{5}+2 c_{3}^{2}-2 c_{3} c_{2}^{2}\right) e_{n}^{3}\right. \\
& +\left(7 c_{3} c_{2}^{3}-8 c_{2} c_{3}^{2}-c_{4} c_{2}^{2}+c_{2} c_{5}+c_{2} \beta c_{3}+5 c_{3} c_{4}+c_{6}\right) e_{n}^{4} \\
& \left.+\cdots+\boldsymbol{O}\left(e_{n}^{9}\right]\right) . \\
f\left[x_{n}, x_{n}, y_{n}\right]= & f^{\prime}(\alpha)\left[c_{2}+2 c_{3} e+\left(3 c_{4}+c_{2} c_{3}\right) e_{n}^{2}+\left(2 c_{2} c_{4}+4 c_{5}+2 c_{3}^{2}\right.\right. \\
& \left.-2 c_{3} c_{2}^{2}\right) e_{n}^{3}+\left(7 c_{3} c_{4}-3 c_{4} c_{2}^{2}-7 c_{2} c_{3}^{2}+4 c_{3} c_{2}^{3}+3 c_{2} c_{5}+5 c_{6}\right) e_{n}^{4} \\
& \left.+\cdots+\boldsymbol{O}\left(e_{n}^{9}\right)\right] .
\end{aligned}
$$

Thus, the estimation of $f^{\prime}\left(z_{n}\right)=p_{f}\left(z_{n}\right)$ described in (9) can be written in terms of $e_{n}$ as:

$$
\begin{aligned}
p_{f}\left(z_{n}\right)= & f^{\prime}(\alpha)\left[1+\left(-2 c_{3} c_{2}^{2}+c_{2} c_{4}+6 c_{2}^{4}+2 \beta c_{2}^{2}\right) e^{4}+\left(-4 c_{2} c_{3}^{2}+40 c_{3} c_{2}^{3}\right.\right. \\
& \left.+4 \beta c_{2} c_{3}+2 c_{3} c_{4}-6 c_{4} c_{2}^{2}+2 c_{2} c_{5}-36 c_{2}^{5}-12 \beta c_{2}^{3}\right) e^{5}+\ldots \\
& +\left(-4 c_{5} c_{3}^{2}+3706 c_{3}^{2} c_{2}^{4}+2 \beta c_{2}^{2}+7 \beta c_{2} c_{6}-75 c_{5} c_{2}^{2} \beta-86 \beta c_{2}^{4}\right. \\
& -102 c_{3} \beta c_{2} c_{4}-787 c_{3}^{3} c_{2}^{2}-5 \beta c_{2} c_{4}+60 \beta c_{3} c_{2}^{2}+3 c_{2}^{2} \beta^{2} c_{3}+380 c_{2}^{3} c_{4} \beta \\
& +464 \beta c_{3}^{2} c_{2}^{2}-1278 c_{3} \beta c_{2}^{4}-4 \beta c_{3} c_{5}+5 c_{2} c_{8}-455 c_{5} c_{2}^{4}+14 c_{6} c_{4} \\
& +96 c_{6} c_{2}^{3}-15 c_{7} c_{2}^{2}-10 c_{3} c_{4}^{2}+184 c_{4}^{2} c_{2}^{2}+1592 c_{4} c_{2}^{5}-4125 c_{3} c_{2}^{6} \\
& +1248 c_{2}^{8}+8 c_{5}^{2}+8 c_{7} c_{3}-3 \beta c_{4}^{2}+642 \beta c_{2}^{6}-54 c_{5} c_{2} c_{4}+309 c_{5} c_{3} c_{2}^{2} \\
& \left.\left.-36 c_{6} c_{2} c_{3}+243 c_{4} c_{2} c_{3}^{2}-1908 c_{4} c_{3} c_{2}^{3}\right) e^{8}+\boldsymbol{O}\left(e_{n}^{9}\right)\right] .
\end{aligned}
$$

Substitute (16)-(20) into $x_{n+1}$ in (10) to obtain that

$$
\begin{aligned}
x_{n+1}= & \alpha+\left(-c_{4} c_{3} c_{2}^{2}+9 c_{2}^{7}+3 c_{4} c_{2}^{4}+c_{3}^{2} c_{2}^{3}-6 c_{3} c_{2}^{5}+6 \beta c_{2}^{5}+c_{2}^{3} \beta^{2}\right. \\
& \left.-2 \beta c_{3} c_{2}^{3}+\beta c_{4} c_{2}^{2}\right) e_{n}^{8}+\boldsymbol{O}\left(e_{n}^{9}\right) .
\end{aligned}
$$

Therefore,

$$
\begin{aligned}
e_{n+1}= & \left(-c_{4} c_{3} c_{2}^{2}+9 c_{2}^{7}+3 c_{4} c_{2}^{4}+c_{3}^{2} c_{2}^{3}-6 c_{3} c_{2}^{5}+6 \beta c_{2}^{5}+c_{2}^{3} \beta^{2}-2 \beta c_{3} c_{2}^{3}\right. \\
& \left.+\beta c_{4} c_{2}^{2}\right) e_{n}^{8}+\boldsymbol{O}\left(e_{n}^{9}\right) .
\end{aligned}
$$

This proves that the order of convergence of the family (10) is eight. This completes the proof. 


\section{Numerical Examples}

In this section, we check for different numerical examples the effectiveness and performance of the family of iterative methods proposed in this paper. Specifically, taking $\beta=0$ in (10), we have:

$$
\begin{gathered}
y_{n}=x_{n}-\frac{f\left(x_{n}\right)}{f^{\prime}\left(x_{n}\right)} \\
z_{n}=x_{n}+\frac{f\left(x_{n}\right)+f\left(y_{n}\right)}{f^{\prime}\left(x_{n}\right)}-2 \frac{f^{2}\left(x_{n}\right)}{f^{\prime}\left(x_{n}\right)\left(f\left(x_{n}\right)-f\left(y_{n}\right)\right)}, \\
x_{n+1}=z_{n}-\frac{f\left(z_{n}\right)}{p_{f}\left(z_{n}\right)} .
\end{gathered}
$$

Taking $\beta=-1$, we have

$$
\begin{gathered}
y_{n}=x_{n}-\frac{f\left(x_{n}\right)}{f^{\prime}\left(x_{n}\right)} \\
z_{n}=x_{n}-2 \frac{f^{2}\left(x_{n}\right)}{f^{\prime}\left(x_{n}\right)\left(f\left(x_{n}\right)-f\left(y_{n}\right)\right)}+\left(\frac{f\left(x_{n}\right)}{f^{\prime}\left(x_{n}\right)}+\frac{f^{\prime}\left(x_{n}\right) f\left(y_{n}\right)}{f^{2}\left(x_{n}\right)+f^{\prime 2}\left(x_{n}\right)}\right), \\
x_{n+1}=z_{n}-\frac{f\left(z_{n}\right)}{p_{f}\left(z_{n}\right)} .
\end{gathered}
$$

We compare our methods (23) and (24) with the eighth-order iterative method of Mir et al. (MM) in [12] which is defined by:

$$
\begin{gathered}
y_{n}=x_{n}-\frac{f\left(x_{n}\right)}{f^{\prime}\left(x_{n}\right)} \\
z_{n}=y_{n}-\frac{h\left(y_{n}\right)}{1-\left(h\left(y_{n}\right)\right)^{2}} \\
x_{n+1}=z_{n}-\left(y_{n}-z_{n}\right) \frac{f\left(z_{n}\right)}{f\left(y_{n}\right)-2 f\left(z_{n}\right)},
\end{gathered}
$$


where

$$
h\left(y_{n}\right)=\frac{2 f\left(y_{n}\right)}{f^{\prime}\left(y_{n}\right)+\sqrt{\left(f^{\prime}\left(y_{n}\right)\right)^{2}+4\left(f\left(y_{n}\right)\right)^{2}}},
$$

and the three eighth-order iterative methods of Wang and Liu (WLM) in [19] which are given as comes next:

$$
\begin{gathered}
y_{n}=x_{n}-\frac{f\left(x_{n}\right)}{f^{\prime}\left(x_{n}\right)} \\
z_{n}=x_{n}-\frac{f\left(x_{n}\right)}{f^{\prime}\left(x_{n}\right)} \frac{f\left(x_{n}\right)-f\left(y_{n}\right)}{f\left(x_{n}\right)-2 f\left(y_{n}\right)} \\
x_{n+1}=z_{n}-\frac{f\left(z_{n}\right)}{f^{\prime}\left(x_{n}\right)}\left[\frac{1}{2}+\frac{5\left(f\left(x_{n}\right)\right)^{2}+8 f\left(x_{n}\right) f\left(y_{n}\right)+2\left(f\left(y_{n}\right)\right)^{2}}{5\left(f\left(x_{n}\right)\right)^{2}-12 f\left(x_{n}\right) f\left(y_{n}\right)}\left(\frac{1}{2}+\frac{f\left(z_{n}\right)}{f\left(y_{n}\right)}\right)\right],
\end{gathered}
$$

and

$$
\begin{gathered}
y_{n}=x_{n}-\frac{f\left(x_{n}\right)}{f^{\prime}\left(x_{n}\right)} \\
z_{n}=x_{n}-\frac{f\left(x_{n}\right)}{f^{\prime}\left(x_{n}\right)} \frac{f\left(x_{n}\right)-f\left(y_{n}\right)}{f\left(x_{n}\right)-2 f\left(y_{n}\right)}, \\
x_{n+1}=z_{n}-\frac{f\left(z_{n}\right)}{f^{\prime}\left(x_{n}\right)}\left\{\frac{5\left(f\left(x_{n}\right)\right)^{2}-2 f\left(x_{n}\right) f\left(y_{n}\right)+\left(f\left(y_{n}\right)\right)^{2}}{5\left(f\left(x_{n}\right)\right)^{2}-12 f\left(x_{n}\right) f\left(y_{n}\right)}\right. \\
\left.+\left[1+\frac{4 f\left(y_{n}\right)}{f\left(x_{n}\right)}\right] \frac{f\left(z_{n}\right)}{f\left(y_{n}\right)}\right\}
\end{gathered}
$$

and

$$
\begin{gathered}
y_{n}=x_{n}-\frac{f\left(x_{n}\right)}{f^{\prime}\left(x_{n}\right)} \\
z_{n}=x_{n}-\frac{f\left(x_{n}\right)}{f^{\prime}\left(x_{n}\right)} \frac{4 f^{2}\left(x_{n}\right)-5 f\left(x_{n}\right) f\left(y_{n}\right)-f^{2}\left(y_{n}\right)}{4 f^{2}\left(x_{n}\right)-9 f\left(x_{n}\right) f\left(y_{n}\right)},
\end{gathered}
$$




$$
x_{n+1}=z_{n}-\frac{f\left(z_{n}\right)}{f^{\prime}\left(x_{n}\right)}\left[1+4 \frac{f\left(z_{n}\right)}{f\left(x_{n}\right)}\right]\left[\frac{8\left(f\left(y_{n}\right)\right)}{4 f\left(x_{n}\right)-11 f\left(y_{n}\right)}+1+\frac{f\left(z_{n}\right)}{f\left(y_{n}\right)}\right] .
$$

The test functions and their roots, found up to the 32 th decimal places, are as follows:

$$
\begin{array}{ll}
\text { Example } & \text { the approximate zero } \alpha \\
f_{1}(x)=\sin x-\frac{x}{2}, & 1.8954942670339809471440357380936, \\
f_{2}(x)=x^{3}-10, & 2.1544346900318837217592935665193, \\
f_{3}(x)=e^{-x}+\cos x, & 1.7461395304080124176507030889538, \\
f_{4}(x)=\sin ^{2} x-x^{2}+1, & 1.4044916482153412260350868177869, \\
f_{5}(x)=x e^{-x}-0.1, & .11183255915896296483356945682027 .
\end{array}
$$

\begin{tabular}{|c|c|c|c|c|c|c|}
\hline & MM (25) & WLM $(26)$ & WLM (27) & WLM (28) & method (23) & Method (24) \\
\hline \multicolumn{7}{|c|}{$f_{1}(x)$, initial guess $x_{0}=2.0$} \\
\hline & 3 & 3 & & 3 & 3 & 3 \\
\hline$\left|f\left(x_{n}\right)\right|$ & $5.3591 \mathrm{e}-327$ & $4.0000 \mathrm{e}-400$ & $4.0000 \mathrm{e}-400$ & $4.0000 \mathrm{e}-400$ & $4.0000 \mathrm{e}-400$ & $4.0000 \mathrm{e}-400$ \\
\hline$\delta$ & $6.5433 \mathrm{e}-327$ & $1.0000 \mathrm{e}-399$ & $1.0000 \mathrm{e}-399$ & 0 & $1.0000 \mathrm{e}-399$ & 0 \\
\hline \multicolumn{7}{|c|}{$f_{2}(x)$, initial guess $x_{0}=2$} \\
\hline & 3 & 3 & 3 & 3 & 3 & 3 \\
\hline$\left|f\left(x_{n}\right)\right|$ & $3.0000 \mathrm{e}-399$ & $3.0000 \mathrm{e}-399$ & $3.0000 \mathrm{e}-399$ & $3.0000 \mathrm{e}-399$ & $1.0000 \mathrm{e}-398$ & $3.0000 \mathrm{e}-399$ \\
\hline & 0 & 0 & 0 & 0 & $1.0000 \mathrm{e}-399$ & 0 \\
\hline \multicolumn{7}{|c|}{$f_{3}(x)$, initial guess $x_{0}=2$} \\
\hline & 3 & 3 & 3 & 3 & 3 & 3 \\
\hline$\left|f\left(x_{n}\right)\right|$ & $6.2529 \mathrm{e}-337$ & $5.0000 \mathrm{e}-400$ & $5.0000 \mathrm{e}-400$ & $5.0000 \mathrm{e}-400$ & $5.0000 \mathrm{e}-400$ & $5.0000 \mathrm{e}-400$ \\
\hline$\delta$ & $5.3945 \mathrm{e}-336$ & 0 & 0 & 0 & 0 & 0 \\
\hline \multicolumn{7}{|c|}{$f_{4}(x)$, initial guess $x_{0}=1.3$} \\
\hline$n$ & 3 & 3 & 3 & 3 & 3 & 3 \\
\hline$\left|f\left(x_{n}\right)\right|$ & $5.5002 \mathrm{e}-392$ & $1.0000 \mathrm{e}-399$ & $1.0000 \mathrm{e}-399$ & $1.7000 \mathrm{e}-399$ & $1.0000 \mathrm{e}-399$ & $1.0000 \mathrm{e}-399$ \\
\hline$\delta$ & $2.2156 \mathrm{e}-392$ & 0 & 0 & $1.0000 \mathrm{e}-399$ & 0 & 0 \\
\hline \multicolumn{7}{|c|}{$f_{5}(x)$, initial guess $x_{0}=0.2$} \\
\hline$n$ & 3 & 3 & 3 & 3 & 3 & 3 \\
\hline$\left|f\left(x_{n}\right)\right|$ & 0 & 0 & 0 & 0 & 0 & 0 \\
\hline & 0 & 0 & 0 & 0 & 0 & 0 \\
\hline
\end{tabular}

All computations were done using MATLAB 7.6 with 400 digit floating arithmetic $(\mathrm{VPA}=400)$. The following criteria

$$
\left|x_{n}-x_{n-1}\right|<\varepsilon \text { and }\left|f\left(x_{n}\right)\right|<\varepsilon,
$$

Table 1: Comparison of various iterative methods of the same order of convergence under the same stopping criteria 
are used for stopping computer programmes. Displayed in Table 1 are the number of iterations $n$, such that the stopping criteria are satisfied, where $\varepsilon$ is taken to be $10^{-300}$, the value of $\left|f\left(x_{n}\right)\right|$ after the required iterations. Moreover, displayed is the distance of two consecutive approximations $\delta=\left|\left(x_{n}-x_{n-1}\right)\right|$.

\section{Conclusions}

In this paper, a new one parameter family of iterative methods with eighthorder of convergence for solving nonlinear equations is presented and analyzed. This new proposed family is optimal since its efficiency index is $8^{1 / 4} \approx 1.6818$. The convergence analysis of the new family is also considered. Several numerical examples are presented to illustrate the efficiency and accuracy of our family. From Table (1), we observe that our family of iterative methods is comparable with all the methods cited in the table.

\section{References}

[1] W. Bi, H. Ren, Q. Wu, Three-step iterative methods with eighth order convergence for solving nonlinear equations, J. Comput. Appl. Math., 225 (2009), 105-112.

[2] C. Chun, iterative method improving Newton's method by decomposition methods, Comput Math. Appl., 50 (2005), 1559-1568.

[3] C. Chun, A family of composite fourth-order iterative methods for solving nonlinear equations, Appl. Math. Comput., 187 (2007), 951-956.

[4] C. Chun-Mei and F. Gao, A new numerical methods for solving nonlinear equations, Int. Math. Forum, 3 (2008), 1437-1443.

[5] A. Cordero, J. L. Hueso, E. Martines and J. R. Torregrosa, A family of iterative methods with sixth and seventh order convergence for nonlinear equations, Math. Comput. Model, 52 (2010), 1490-1496..

[6] J. Kou, X. Wang and S. Sun, Some new root-finding methods with eighthorder convergence, Bull. Math. Soc. Sci. Math. Roumanie, 53 (2010), 133143.

[7] J. Kou, Y. Li, X. Wang, Some modifications of Newton's method with fifth order convergence, J. Comput.Appl. Math., 209 (2007), 146-152. 
[8] H. T. Kung, J.F. Traub, Optimal order of one-point and multipoint iteration,J. Assoc. Comput, March 21 (1974), 643-651.

[9] L. Liu, X. Wang, Eighth-order methods with high efficiency index for solving nonlinear equations, Appl. Math. Comput., 215 (2010), 3449-3454.

[10] B. Neta, M. S. Petkovic, Construction of optimal order nonlinear solvers using inverse interpolation, Appl. Math. Comput.,217 (2010), 2448-2455.

[11] M. A. Noor, W. A. Khan, K. I. Noor and Eisa Al-Said, Higher-order iterative methods free from second derivative for solving nonlinear equations, Int. J. Phys. Sci., Vol. 6 (8) (2011), 1887-1893.

[12] Nazir A. Mir, Naila Rafiq and Saima Akram, An Efficient Three-Step Iterative Method For Non-Linear Equations,Int. J. Math. Analysis, Vol. 3, No. 40 (2009), 1989-1996.

[13] J. R. Sharma and R. Sharma, A new family of modified Ostrowski's methods with accelerated eighth order convergence, Numer. Algorithms, 54 (2010), 445-458.

[14] L. D. Petkovic, M.S. Petkovic, J. Dzuniz, A class of three-point root-solver of optimal order of convergence, Appl. Math. Comput., 216 (2010), 671676.

[15] H. I. Siyyam, An iterative method with fifth-order convergence for nonlinear equations,, Appl. Math. Sci., 3 (2009), 2041-2053.

[16] F. Soleimani and F. Soleymani, Computing simple roots by a sixth-order iterative method, Int. J. Pure. Appl. Math., 69 (2011), 41-48.

[17] F. Soleymani and V. Hosseniabadi, New third and sixth-order derivativefree techniques for nonlinear equations, J. Math. Research, 3 (2011), 107112.

[18] P. Wang, A third-order family of Newton-like iteration methods for solving nonlinear equations, J. Numer. Math. Stoch., 3 (2011), 13-19.

[19] X. Wang and L. Liu, New eighth-order iterative methods for solving nonlinear equations, J. Comput. Appl. Math., 234 (2010), 1611-1620.

[20] J.F. Traub, Iteraive Methods for the Solution of Equations, Chelsea publishing company, New York (1982). 
